\title{
Changes in the power spectrum of electrical signals in maize leaf induced by osmotic stress
}

\author{
ZHANG XiaoHui ${ }^{1}$, YU NingMei $^{1 *}, \mathrm{XI} \mathrm{Gang}^{2}$ \& MENG XiaoLi ${ }^{2}$ \\ ${ }^{1}$ Faculty of Automation and Information Engineering, Xi' an University of Technology, Xi'an 710048, China; \\ ${ }^{2}$ Department of Applied Physics, Xi'an University of Technology, Xi'an 710048, China
}

Received April 11, 2011; accepted September 21, 2011

\begin{abstract}
To quantify the characteristics of the power spectrum of plant electrical signals, we defined the following concepts: spectral edge frequency (SEF), spectral center frequency (SCF), power index (PI) and power spectral entropy (PSE). These parameters were used to examine and quantify changes in the power spectrum of electrical signals in maize leaves under osmotic stress. In the absence of osmotic stress, the SEF of the electrical signal in maize leaves was approx. $0.2 \mathrm{~Hz}$ and the SCF was approx. $0.1 \mathrm{~Hz}$. The electrical signal in maize leaves was mainly a slow wave signal with a frequency of $0-0.1 \mathrm{~Hz}$. After $2 \mathrm{~h}$ osmotic stress, the SEF and SCF of the electrical signal increased to higher frequencies. The proportion of the fast wave frequency also increased to 0.1-0.2 Hz, resulting in a dramatic increase in PSE. We also found that the changes in PSE and SCF were significantly correlated during osmotic stress. We propose that the changes in the PSE and SCF in maize leaves can be used as a sensitive signal indicating water deficit in leaf cells under osmotic stress. Thus, measurement of SCF or PSE of electrical signals in maize leaves could be used to develop early warning and rapid diagnosis techniques for the water demands of plants.
\end{abstract}

plant electrical signal, power spectrum, osmotic stress, maize

Citation: Zhang X H, Yu N M, Xi G, et al. Changes in the power spectrum of electrical signals in maize leaf induced by osmotic stress. Chin Sci Bull, 2012, 57: 413-420, doi: 10.1007/s11434-011-4820-5

In recent years, there has been increasing interest in plant electrical signals. This is because plant electrical signals, chemical signals (phytohormones), mechanical signals, and water signals constitute the plant information system $[1,2]$. Electrical signals are not only able to rapidly transmit information over long distances within the plant body $[1,3,4]$, but also affect major physiological processes such as respiratory metabolism, photosynthesis, moisture absorption, and stomatal conductance [1,5-7]. It is now generally considered that plants undergoing normal growth and development show fluctuations of potential. On one hand, this reflects the flow of information throughout the tissues and organs of the plant, and on the other, it reflects a level of direct control and regulation of responses to environmental stimuli $[3,4$, 8-10].

*Corresponding author (email: yunm@xaut.edu.cn)
At present, studies on plant electrical signals focus on two main aspects. One aspect is the production, transmission, reception, and transformation mechanisms of plant electrical signals. The purpose of such studies is to unravel the internal information network of the plant and to understand how plants respond to environmental stimuli; that is, to clarify aspects of plant neurobiology [1,4,11-13]. The other research focus is to understand how plant electrical signals convey information. Plant electrical signals respond quickly to changes in light intensity, osmotic pressure, temperature, mechanical damage, mechanical stimulation, water availability, chemical compounds (i.e., plant growth stimulators or herbicides), and salt. The changes in electrical signals occur earlier than other physiological and morphological changes $[12,14,15]$. Thus, reading and analysis of plant electrical signals could provide an early warning system, and could enable real-time evaluation of changes in 
the plant state in response to environmental stress [16]. Thus, accurate and reliable phyto-monitoring techniques could be developed based on plant electrical signals.

Previous studies on phyto-monitoring techniques based on plant electrical signals showed that plants had a rapid reaction to mechanical stimuli, such as pruning [17-19]. Therefore, remote real-time monitoring of the plant electrical signals [16] could provide an effective tool for early detection of insect damage, strong wind damage, or damage from human activities.

Other studies found that plants responded very rapidly to fluctuations in soil water content, and the rapid conducting of the electrical signal between roots and leaves affected stomatal regulation $[3,8]$, photosynthesis, and respiration $[7,20]$. Accordingly, water deficit and its regulation in maize leaves could be estimated from the difference between the magnitudes of the electrical potential in leaves and roots of maize in conditions of soil drought.

For phyto-monitoring techniques based on the plant electrical signal, the key problem to be solved is how to determine and measure the characteristic parameters of these signals to achieve an accurate quantitative analysis. The correlation between the electrical signal and the environmental stimulus can be studied to interpret plant responses; that is, to understand "the speaking-plant" [21]. However, the only attribute of the electrical signal that can be measured (and therefore, interpreted) is the amplitude of the potential fluctuation of the signal. Because the plant electrical signal is random and its amplitude is constantly changing, the changes of amplitude are hard to measure accurately, which leads to difficulties in practical applications.

The power spectrum of the electrical signal is the distribution of signal power over different frequencies. Thus, it characterizes the frequency characteristics of the signal. The power spectrum distribution of the plant electrical signal is related to the transmembrane transportation of various ions, changes in membrane potential, and electrical coupling between cells. It is the result of interactions and coordination among cells [22]. There is a particular power spectrum distribution of the electrical signal when plants are in a particular life stage. When the plant is subjected to interference of some kind, the power spectrum distribution will change. Because the plant electrical signal has a stable power spectrum distribution when the plant is in a particular state, there is a clear advantage to evaluating changes in the plant state according to changes of characteristic parameters of the power spectrum, rather than the amplitude of the electrical signal. The aim of this study was to establish an index system to describe the characteristics of the power spectrum. This study is preliminary research on the changes in the characteristic parameters of the power spectrum of the electrical signal in maize leaves under osmotic stress. We hope to develop this research into early warning and diagnosis methods for evaluating the water requirements of plants.

\section{Materials and method}

\subsection{Material and osmotic stress}

Maize seeds (Shannong 226) were provided by the Agricultural Institute of the Northwest Agriculture and Forestry University. Seeds were germinated on wet filter paper at a incubator at a temperature of $30^{\circ} \mathrm{C}$ constant temperature in a germination box. Once germinated, seeds were transplanted into a pot which filled with soil. The soil contained all nutrients essential for plant growth and was composed of 50\% peat, $35 \%$ clay and $15 \%$ humus ( $\mathrm{pH} 6.0$ ), and grown under a light intensity of $60 \mu \mathrm{mol} \mathrm{m} \mathrm{s}^{-2} \mathrm{~s}^{-1}$ provided by mercury halide lamps in a $14 / 10 \mathrm{~h}$ light/dark period, at a watering cycle of $3 \mathrm{~d}$ with $1 \% \mathrm{KH}_{2} \mathrm{PO}_{4}$ solution. To apply osmotic stress, $10 \%$ PEG6000 solution was added to the soil when the maize plants were at the 3-leaf stage. The electrical signals of maize leaves were measured after various periods of time under osmotic stress.

\subsection{Measuring instrument}

We used the BL-420E biological function experiment system (designed by Chengdu TME Technology Co, Ltd.) to measure electrical signals. The input impedance is $400 \mathrm{M} \Omega$, and every input channel is photoelectrically isolated, which decreases outside interference. The equivalent input noise voltage of the instrument was less than $1 \mu \mathrm{V}$. The measuring system is shown in Figure 1. The constant temperature control system, shielding box, and measuring instrument were connected to each other. The sampling frequency was $5 \mathrm{kHz}$, the frequency of the low-pass filter was $500 \mathrm{~Hz}$, and that of the high-pass filter was $0.053 \mathrm{~Hz}$. The unit of electrical potential energy was $\mu \mathrm{V}$, the unit of time was second. During sampling, the temperature of the environment was $20^{\circ} \mathrm{C}$ and the humidity was $40 \%-50 \%$.

The electrode was a high-sensitivity silver AMBU ECG electrode. Before the experiment, a test of the electrodes showed that they detected $0-50 \mathrm{~Hz}$ signals with fluctuations of less than $0.5 \%$, demonstrating high electrode stability. To measure the plant electrical signal, the A electrode was placed on the front surface of the leaf and the B electrode on the back (leaf length, $25 \mathrm{~cm}$; leaf width, $2 \mathrm{~cm}$ ). The distance between two electrodes was $15 \mathrm{~cm}$. The $\mathrm{C}$ electrode was connected to the copper sheet, which was in the soil. The measuring electrode was connected to the signal measurement system, and the electrical signal was monitored and recorded by a computer. The environment temperature was regulated by the constant temperature controller.

\subsection{Signal processing algorithms}

The plant electrical signal is a non-steady and non-linear random signal. In this study, we used Matlab software as the research platform. The electrical signal of maize was analyzed 


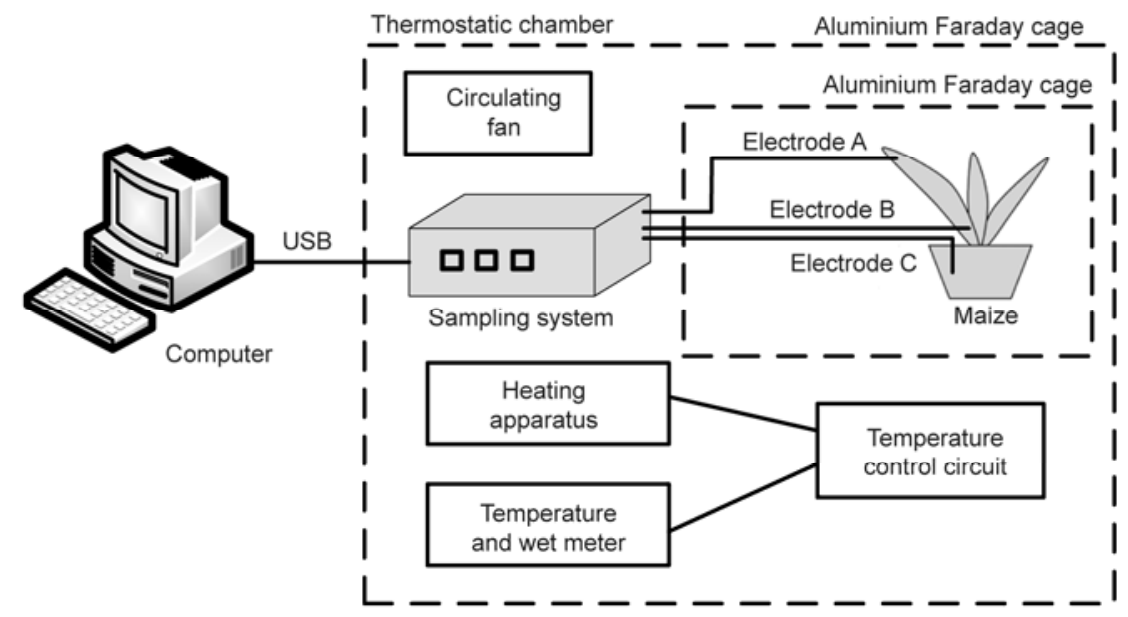

Figure 1 Schematic diagram of experimental device.

and disposed with the wavelet soft threshold de-noising and Discrete Fast Fourier Transform (DFFT).

(i) Wavelet transformation. For the finite length signal $f(t)$ with noise, a suitable wavelet function is selected as the wavelet transform:

$$
\left(W_{\psi} f\right)(a, b)=\left\langle f, \psi_{a, b}\right\rangle=|a|^{-1 / 2} \int_{-\infty}^{+\infty} f(t) \psi^{*}\left(\frac{t-b}{a}\right) \mathrm{d} t,
$$

where $a, b \in \mathrm{R}, a \neq 0, a$ is scaling factor, $b$ is a displacement factor, and $\psi^{*}$ is the conjugate operation of $\psi$.

The wavelet coefficients are processed by soft threshold in the wavelet transforming domain. When selecting the threshold, the standard deviation of original signal's noise must be estimated. In this study, we used eq. (2) to estimate noise deviation.

$$
\sigma=\frac{\operatorname{median}\left(\left|d_{j}(k)\right|\right)}{0.6745}\left(k=0,1, \cdots, 2^{j}-1\right),
$$

where $j$ is the wavelet decomposition scale, and median is the Matlab command. The Donoho threshold estimation formula [14] is then used to calculate the threshold.

$$
\operatorname{thr}=\sigma \sqrt{2 \log (n)},
$$

where $n$ is the length of signal.

Then, wavelet coefficients are determined by using the wavelet de-noising soft threshold rules. When the absolute value of wavelet coefficients is less than the threshold, the wavelet coefficient is zero. Otherwise, the wavelet coefficient is the value of subtracting the threshold from the absolute value of wavelet coefficients which is greater than the threshold. This is summarized in eq. (4):

$$
d_{j, \mathrm{thr}}=\left\{\begin{array}{l}
0,\left|d_{j}\right|<\mathrm{thr}, \\
\operatorname{sgn}\left(d_{j}\right)\left(\left|d_{j}\right|-\mathrm{thr}\right),\left|d_{j}\right| \geqslant \mathrm{thr},
\end{array}\right.
$$

where $d_{j, \text { thr }}$ is the value of the wavelet coefficient processed by the threshold. Finally, the signal $f(t)$ is obtained with the inverse wavelet transform as follows:

$$
f(t)=\frac{1}{C_{\psi}} \int_{-\infty}^{+\infty} \int_{-\infty}^{+\infty}\left[\left(W_{\psi} f\right)(a, b)\right] \psi\left(\frac{t-b}{a}\right) \frac{\mathrm{d} a}{a^{2}} \mathrm{~d} b,
$$

where $C_{\psi}=\int_{-\infty}^{+\infty}|\omega|^{-1}|\hat{\psi}(\omega)|^{2} \mathrm{~d} \omega<\infty, \quad \hat{\psi}(\omega)$ is the Fourier transform of $\psi(t)$, and $f(t)$ is the result with inverse wavelet transform and reconstruction.

(ii) Power spectrum analysis. For the signal $x(n)$ with a finite length of time and a discrete sequence, the spectrum characteristics were obtained with the following equation [23]:

$$
X\left(\mathrm{e}^{j \omega}\right)=\sum_{n=-\infty}^{n=+\infty} x(n) \mathrm{e}^{-j \omega n}
$$

where $X\left(\mathrm{e}^{j \omega}\right)$ was obtained by discrete time Fourier transformation of the sequence signal $x(n)$, and the frequency-domain characteristics of the original $x(n)$ are obtained through $X\left(\mathrm{e}^{j \omega}\right)$.

The characteristic parameters of the power spectrum analysis in this paper are defined as follows:

(1) Spectral edge frequency (SEF): The power of the signal between $0 \mathrm{~Hz}$ and the SEF accounts for $95 \%$ of the total power. The unit of SEF is Hz. It gives the frequency range of the signal distribution.

(2) Spectral center frequency (SCF), which is the center of the signal power distribution with frequency, derived using the following formula:

$$
f_{\mathrm{g}}=\frac{\sum_{f=f_{1}}^{f_{2}}[P(f) \times f]}{\sum_{f=f_{1}}^{f_{2}} P(f)},
$$


where $f_{\mathrm{g}}$ is the spectral center frequency and the frequency ranges between $f_{1}$ and $f_{2}, P(f)$ is signal power, and $f$ is the value of the frequency.

(3) Power index (PI): PI includes $\delta$ index, $\theta$ index, $\alpha$ in$\operatorname{dex}$, and $\beta$ index. These separately express the total power in the frequency range of $\delta, \theta, \alpha$ and $\beta$, where the frequency range of $\delta, \theta, \alpha$ and $\beta$ are defined as follows: $\delta(0-0.1 \mathrm{~Hz})$, $\theta(0.1-0.2 \mathrm{~Hz}), \alpha(0.3-0.4 \mathrm{~Hz}), \beta(0.5-\infty \mathrm{Hz})$.

(4) Power spectral entropy (PSE), which reflects the degree of complexity of the signal defined as follows:

$$
H_{f}=-\sum p_{f} \ln p_{f},
$$

where the subscript $f$ is frequency, and the subscript $p_{f}$ is the percentage of power at the frequency $f$ in the entire spectrum of power.

\section{Results}

\subsection{Time domain characteristics of electrical signal in maize leaf under osmotic stress}

Plant electrical signals are very weak, and are easily masked by background noise. Although the measuring instrument and the shield method used in this study can reduce external interference, there is still some background noise. To obtain the most accurate power spectrum of signals in maize leaves, we used a wavelet transform method to de-noise the original signal. The time domain waveform of the electrical signal in maize leaf is shown in Figure 2(a)-(d). This waveform was decomposed through a db5 wavelet at seven scales, soft threshold de-noised, and reconstructed. The electrical signals were sampled separately at $0,2,4$, and $6 \mathrm{~h}$ of osmotic stress.

\subsection{Power spectrum analysis of electrical signal in leaf of maize}

Fast Fourier transform was used to analyze the spectrum of the electrical signal in maize leaves. The power spectrum distributions at various intervals under osmotic stress are shown in Figure 3.

(i) Changes of SEF and SCF of electrical signal spectrum. To quantitatively explain the changes in the electrical signal spectrum of maize under different osmotic conditions, the changes of SEF and SCF of the electrical signal spectrum of maize leaves were calculated at various time points during osmotic stress. As shown in Figure 4, the SEF of the electrical signal of maize leaves was about $0.2 \mathrm{~Hz}$ in the absence of osmotic stress, but rapidly increased by $2 \mathrm{~h}$ of osmotic stress. This result shows that the distribution of the power spectrum distribution gradually extends to a higher frequency after $2 \mathrm{~h}$ of osmotic stress, but decreases after that. The trend in SCF was similar to that of SEF under osmotic stress. The synchronous increase of SCF and SEF shows that the changes in the center of the power spectrum distribution and the range of frequency distribution are essentially the same.
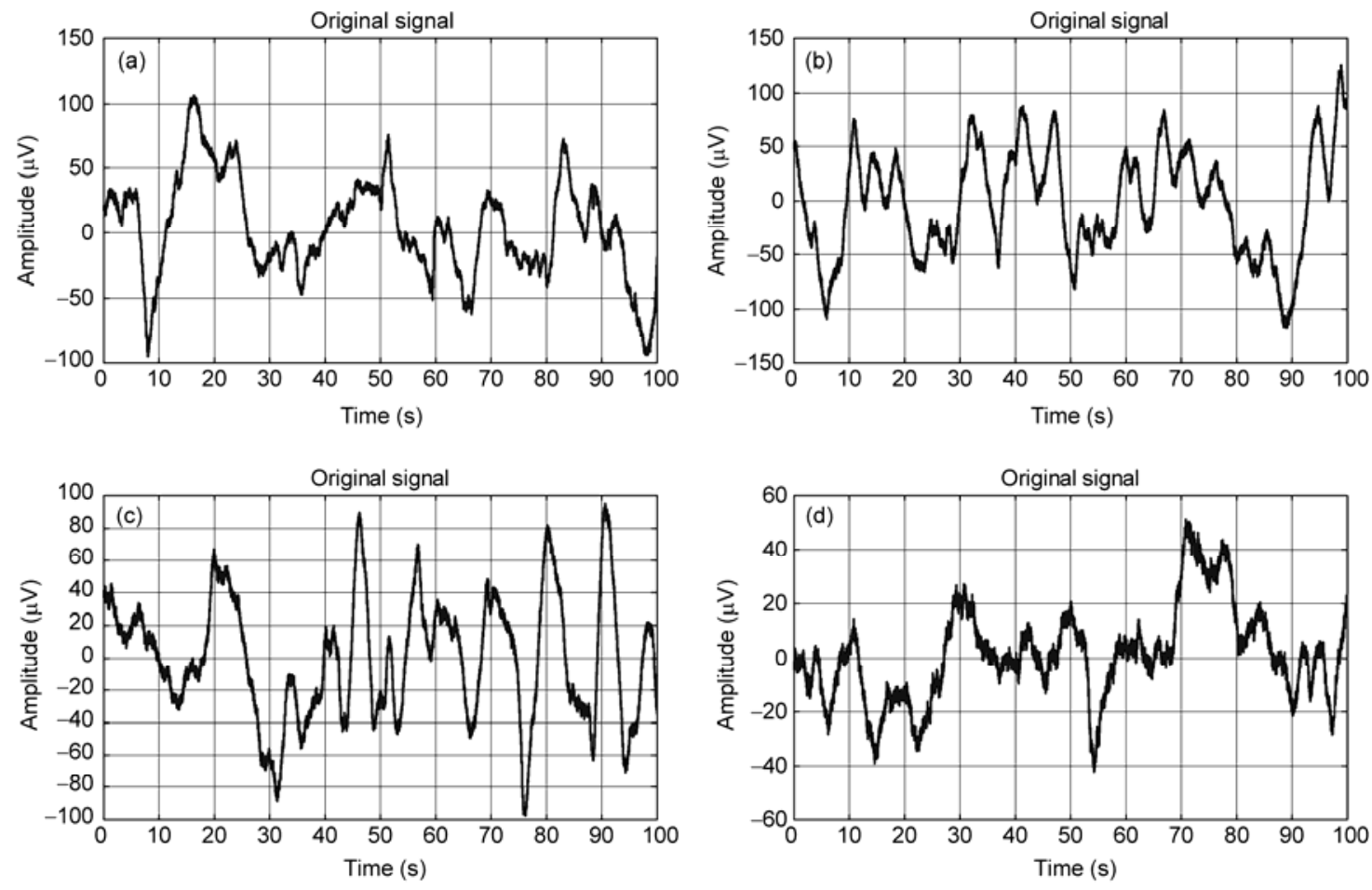

Figure 2 Time domain waveform of electrical signal of maize leaf under osmotic stress. (a) $t=0 \mathrm{~h}$; (b) $t=2 \mathrm{~h}$; (c) $t=4 \mathrm{~h}$; (d) $t=6 \mathrm{~h}$. 

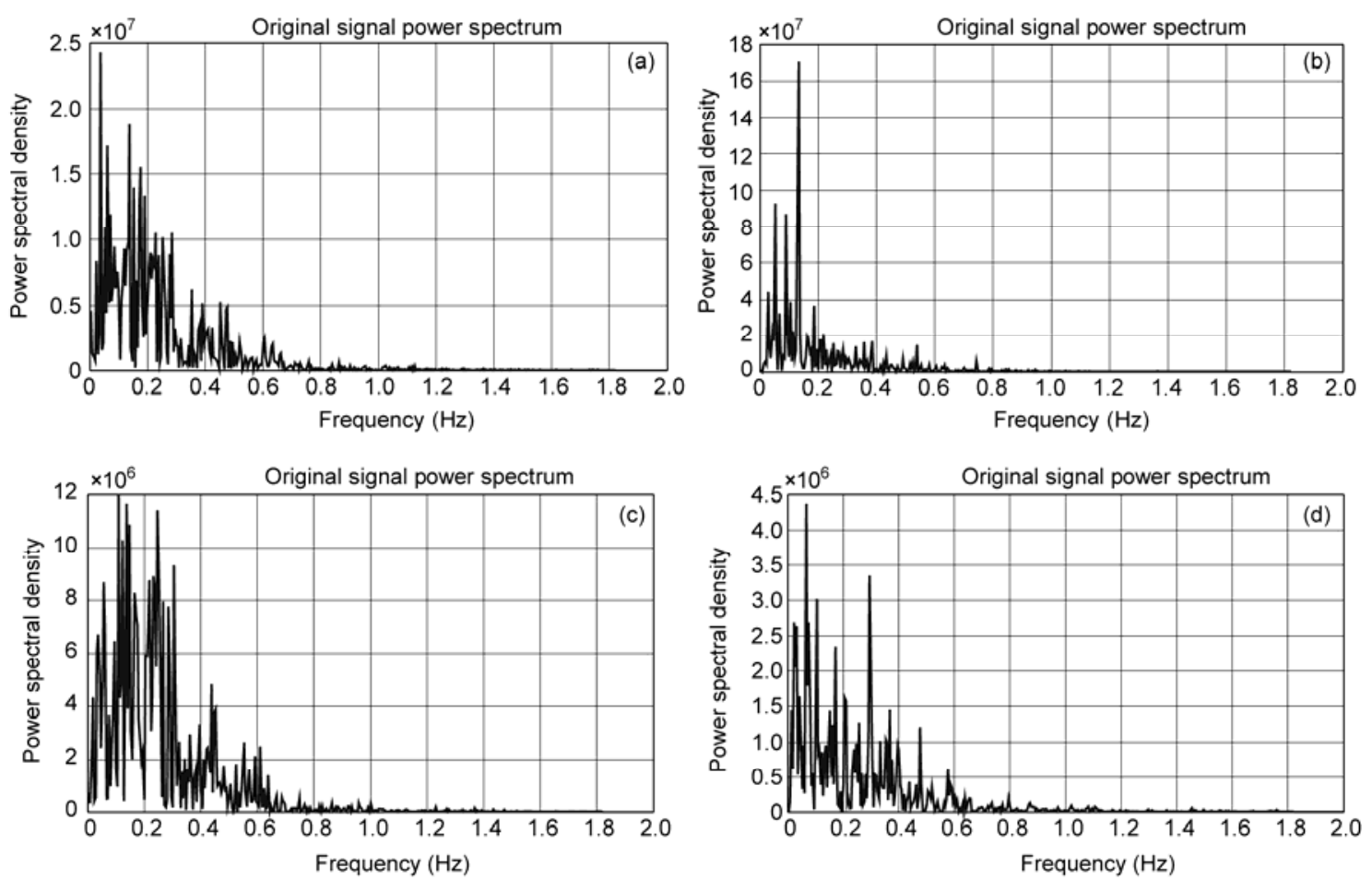

Figure 3 Power spectrum distribution of electrical signals in maize leaf under osmotic stress. (a) $t=0 \mathrm{~h}$; (b) $t=2 \mathrm{~h}$; (c) $t=4 \mathrm{~h}$; (d) $t=6 \mathrm{~h}$.

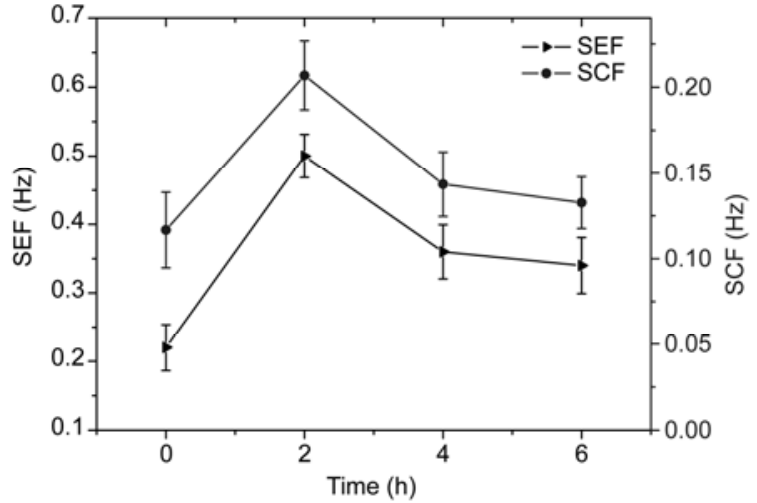

Figure 4 Changes in SEF and SCF of electrical signals over time in maize leaves under osmotic stress.

(ii) Changes of electrical signal PI. Figure 5 shows in details the changes in SCF of the electrical signal in maize leaves. In the absence of osmotic stress $(t=0 \mathrm{~h})$, the frequencies of the electrical signal are mainly distributed at the $\delta$ band. As well, the electrical signal in maize leaves is mainly slow wave signal $(0-0.1 \mathrm{~Hz})$. After $2 \mathrm{~h}$ osmotic stress, the proportion of the $\theta$ band $(0.1-0.2 \mathrm{~Hz})$ had increased, but then decreased between 4 and $6 \mathrm{~h}$ of osmotic stress. The changes in the $\delta$ index showed the opposite trend. During the entire osmotic stress treatment, the $\alpha$ and $\beta$ indices were largely unchanged. If the activation coefficient is defined as $\theta / \delta$, the greater value of $\theta / \delta$ indicates higher cellular activity. The result in Figure 5 shows that cellular activity was greatest after $2 \mathrm{~h}$ of osmotic stress, but returned to its initial

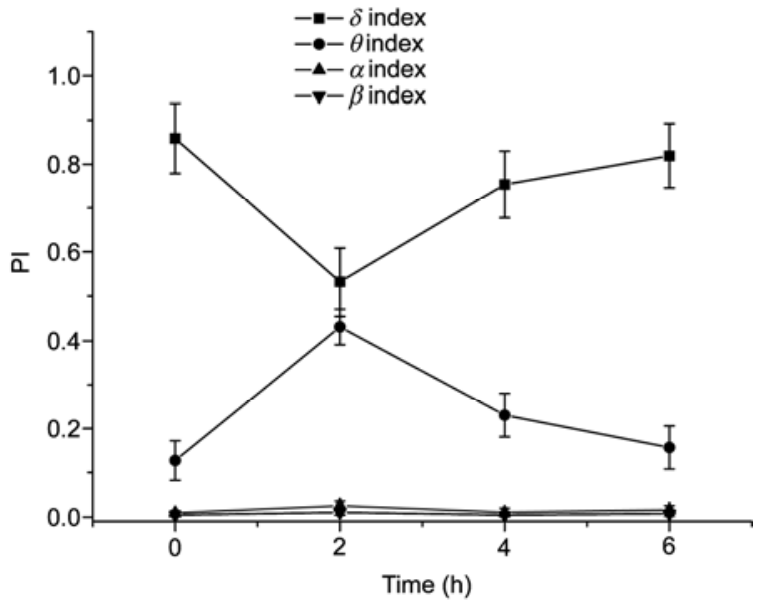

Figure 5 Changes in the electrical signal power index during osmotic stress.

state at 4 and $6 \mathrm{~h}$.

(iii) Changes of power spectrum peak of electrical signal. Figure 6 shows the changes in the corresponding power spectrum peak. The maximum value of the electrical signal in the absence of osmotic stress was $2.43 \times 10^{7} \mathrm{~W} / \mathrm{Hz}$, and the corresponding frequency was $0.03 \mathrm{~Hz}$. After $2 \mathrm{~h}$ of osmotic stress, these values were $1.7 \times 10^{8} \mathrm{~W} / \mathrm{Hz}$ and $0.13 \mathrm{~Hz}$, respectively. After $4 \mathrm{~h}$ of osmotic stress, these values were $1.2 \times 10^{7} \mathrm{~W} / \mathrm{Hz}$ and $0.11 \mathrm{~Hz}$, respectively. After $6 \mathrm{~h}$ of osmotic stress, these values were $4.4 \times 10^{6} \mathrm{~W} / \mathrm{Hz}$ and $0.06 \mathrm{~Hz}$, respectively. From these results, it seems that the power spectrum peak of the electrical signal in maize leaf increases 


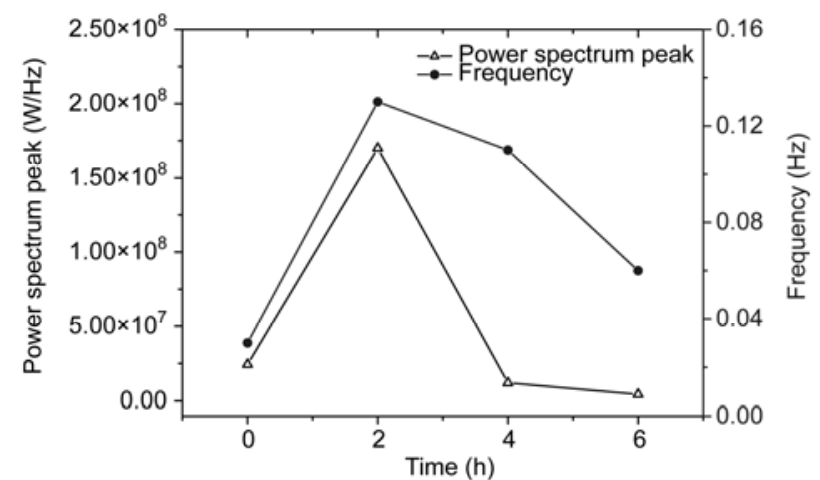

Figure 6 Changes in the power spectrum peak of the electrical signal in maize leaves during osmotic stress.

after $2 \mathrm{~h}$ osmotic stress, then decreases greatly after 4 and $6 \mathrm{~h}$ osmotic stress. This marked increase in the power spectrum peak indicates that it may be produced by action potential (AP), and that the frequency of the AP is $0.13 \mathrm{~Hz}$, which is in $\theta$ band. It appeared that the in SCF was caused by AP.

(iv) Changes in PSE of the electrical signal in maize leaves. The degree of complexity of the electrical signal power spectrum can be expressed by PSE. The changes in the PSE in maize leaves under osmotic stress are shown in Figure 7. The maximum PSE value was obtained after $2 \mathrm{~h}$ osmotic stress. The PSE decreased by $4 \mathrm{~h}$ of osmotic stress, and decreased further, almost to pre-stress levels, at $6 \mathrm{~h}$ of osmotic stress. The trend in PSE was similar to those shown by SEF and SCF.

\section{Discussion}

Plant electrical signals recorded by electrodes are spontaneous and rhythmic electrical activities of clusters of plant cells. When plants are unstimulated, the electrical signal is stable and is designated as the local potential (LP). When plants are stimulated, cells generate action potential (AP)

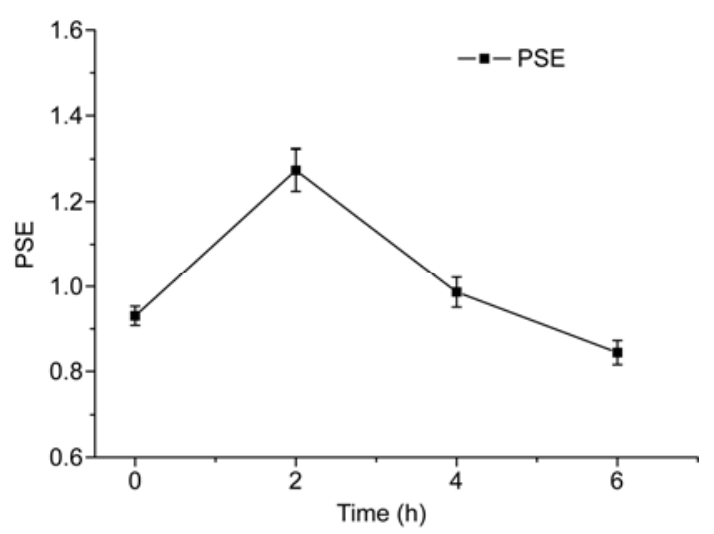

Figure 7 Changes in PSE of the electrical signal in maize leaves during osmotic stress. and variation potential (VP). The AP can be transmitted immediately within tissues and organs at high speed and constant amplitude [1,5,10,24], as long as a certain threshold is reached [12,25]. AP results from transport of ions across membranes and depends on the penetrability of different ions and transient variations in the external environment, electrochemical potential, and the cytoplasm [26-28]. VP is related to transient transfers of membrane potential, but its amplitude and speed decrease over time and with distance from the site of stimulation. According to a previous study [29], weak stimulation generates AP, while strong stimulation generates VP. Most plant cells are electro-active, and thus, are able to release and transmit AP to roots, branches, buds [4], petioles [2], and leaves [30].

As reported by Fromm and Fei [8], arid stimulation of maize roots causes AP, which moves to leaves via sieve tubes of the phloem and closes stomata. Transmission of AP to the leaves disturbs the potential distribution in the leaves, resulting in changes to the distribution of the signal power spectrum. To quantitatively describe the characteristics and changes of the plant electrical signal power spectrum, we propose the concepts of SEF and SCF along with a computational method to interpret these values. The SEF reflects the frequency distribution range of the signal, and the SCF shows the distribution center of the signal frequency. SCF not only reflects the distribution of the signal power spectrum and shows a large component of the spectrum, but also shows the migration of the entire power spectrum under different conditions. To describe the frequency distribution of the signal power in detail, we also defined PI and $\theta / \delta$ to show cellular reactions.

In this study, we observed that the peak value of the leaf electrical signal power spectrum increased dramatically after $2 \mathrm{~h}$ osmotic stress (Figure 6). This increase was due to transmission of AP from the root. As the peak value frequency reached $0.13 \mathrm{~Hz}, \mathrm{SEF}$ and SCF increased to higher frequencies. At the same time, $\delta$ decreased and $\theta$ increased (Figure 5). The increase in the activation coefficient indicates greater cellular activities. It appears that the changes in SCF and $\theta / \delta$ under osmotic stress reflect the responses of leaf cells to osmotic stress.

In actively growing plants, the electrical signals in the time domain curve appear to have random features, and the power spectrum of these signals shows a continuous distribution throughout the frequency domain. The continuous distribution of the power spectrum of the electrical signals in plant leaves reflects the complexity of normal growth. To quantitatively describe the complexity of the signals and the changes in their power spectrum distribution, we propose the use of the power spectral entropy (PSE) of the plant electrical signal (eq. (8)). This is derived from the Shannon entropy theory, and measures the complexity of the power spectrum distribution. It describes the degree of disorder of the time series signal and the structural situation of the time series signal spectrum. The more uniform the distribution of 
the signal energy across all frequencies, that is, the flatter the power spectrum, the more complex the signal [31]. This situation is reflected by high PSE values. Conversely, smaller PSE values reflect a narrower signal power spectrum. If there is an obvious oscillation rhythm in the signal, then the complexity of the signal is low. Figure 7 shows that the PSE of the maize electrical signal significantly increased during osmotic stress, indicating increased complexity of the electrical activity in plant cells. This may be because the AP induced in the root by osmotic stress was transferred to the leaf cells, increasing the amount of information accepted by leaf cells. This can be visualized by comparing the changes of PSE with those of SCF; here, the changes of PSE and SCF were synchronized. This consistent pattern of change in PSE and SCF illustrates that these values mirror the responses of maize leaves to osmotic stress. While they both represent changes in the power spectrum of the electrical signal, the PSE and SCF explain different aspects of these changes.

The findings of this paper are highly significant, because they provide methods to rapidly analyze plants' responses to environmental stimuli. This represents a technological innovation in modern agriculture that can be used for research on water-saving ecology and other subjects. For example, it could be used to gather information about the water demands of crops so that irrigation could be controlled precisely. The foundation for such research is the development of a diagnostic indicator system to measure crop signals in response to water stress, to determine water stress thresholds, and consequently, to achieve the greatest water use efficiency [32]. The proposed indicators, SCF and PSE, can be used to analyze plants signals and to quantify water demand. As well, they can be used as an early warning system for plant water status. Because changes in plant electrical signals occur earlier than other physiological and morphological changes [12,14,15], the changes of SCF or PSE can be used as rapid and accurate indicators of plant water demands. Therefore, these methods can be developed as a new, nondestructive testing technology to acquire information.

\section{Conclusions}

(1) The power spectrum distribution of the plant electrical signal can be quantitatively described by SEF, SCF, and PI. During normal growth of maize, the SEF of the electrical signal in the leaf was approx. $0.2 \mathrm{~Hz}$ and the SCF was approx. $0.1 \mathrm{~Hz}$. After $2 \mathrm{~h}$ of osmotic stress, the values of SEF and SCF rapidly increased, indicating an increase in high frequency signals, a reduction of low frequency signals, and stimulation of cellular activity. After $6 \mathrm{~h}$ of stress, the values SEF and SCF decreased.

(2) The degree of complexity of the plant electrical signal can be quantitatively described by PSE. After $2 \mathrm{~h}$ of osmotic stress, the rapid increase in PSE indicated an increase in the complexity of cellular activity. After $6 \mathrm{~h}$ of osmotic stress, the PSE had returned to a low (pre-stress) level.

(3) The changes in PSE and SCF during osmotic stress are highly relevant, and they can be used as a sensitive signal of water deficit. Both of these parameters reflect regulation of leaf cells that occurs under osmotic stress. These methods have potential applications for monitoring plant responses to environmental change, for evaluating stress resistance, for physiological studies and for ecological/environmental monitoring.

This work was supported by the National Natural Science Foundation of China (50977079) and the Scientific Research Plan Project of Shaanxi Education Department (09JK667).

1 Fromm J, Lautner S. Electrical signals and their physiological significance in plants. Plant Cell Environ, 2007, 30: 249-257

2 Gil P, Gurovich L, Schaffer B, et al. Electrical signaling, stomatal conductance, $\mathrm{ABA}$ and ethylene content in avocado trees in response to root hypoxia. Plant Signal Behav, 2009, 4: 100-108

3 Gil P, Gurovich L, Schaffer B, et al. Root to leaf electrical signaling in avocado in response to light and soil water content. Plant Physiol, 2008, 165: 1070-1078

4 Gurovich L, Hermosilla P. Electrical signaling in fruit trees in response to water applications and light-darkness conditions. Plant Physiol, 2009, 166: 290-300

5 Trebacz K, Dziubinska H, Krol E. Electrical signals in long-distance communication in plants. In: Baluska F, Mancuso S, Volkmann D, eds. Communication in Plants-Neuronal Aspects of Plant Life. Berlin, Heidelberg: Springer-Verlag, 2006. 277-290

6 Koziolek C, Grams T E E, Schreiber U, et al. Transient knockout of photosynthesis mediated by electrical signals. New Phytol, 2003, 161: 715-722

7 Masi E, Ciszak M, Stefano G, et al. Spatiotemporal dynamics of the electrical network activity in the root apex. Proc Natl Acad Sci USA, 2009, 106: 4048-4053

8 Fromm J, Fei H. Electrical signaling and gas exchange in maize plants of drying soil. Sci Plant, 1998, 132: 203-213

9 Volkov A, Adesina T, Markin V, et al. Kinetics and mechanism of Dionaea muscipula trap closing. Plant Physiol, 2008, 146: 694-702

10 Lautner S, Erhard T, Matyssek R, et al. Characteristics of electrical signals in poplar and responses in photosynthesis. Plant Physiol, 2005, 198: 2200-2209

11 Baluska F, Mancuso S, Volkmann D, et al. Root apices as plant command centers: The unique 'brain-like' status of the root apex transition zone. Biol Brat, 2004, 59: 1-13

12 Brenner E D, Stahlberg R, Mancuso S, et al. Plant neurobiology: An integrated view of plant signaling. Sci Trends Plant, 2006, 11: 413-421

13 Trewavas A. Green plants as intelligent organisms. Sci Trends Plant, 2005, 10: 414-419

14 Donoho D L. De-noising by soft-thresholding. IEEE Trans Inform Theory, 1995, 41: 613-627

15 Yan X F, Wang Z Y, Huang L, et al. Research progress on electrical signals in higher plants. Prog Nat Sci, 2009, 19: 531-541

16 Oyarce P, Gurovich L. Evidence for the transmission of information through electrical potentials in injured avocado trees. Plant Physiol, 2011, 168: 103-108

17 Datta P, Palit P. Relationship between environmental factors and diurnal variation of bioelectrical potentials of an intact jute plant. Curr Sci Bangalore, 2004, 87: 680-683

18 Volkov A, Carrell H, Markin V. Biologically closed electrical circuits in Venus flytrap. Plant Physiol, 2009, 149: 1661-1667

19 Wang Z, Leng Q, Huanga L, et al. Monitoring system for electrical 
signals in plants in the greenhouse and its applications. Biosyst Eng, 2009, 103: 1-11

20 Grams T E E, Koziolek C, Lautner S, et al. Distinct roles of electrical and hydraulic signals on the reaction of leaf gas exchange upon reirrigation in Zea mays L. Plant Cell Environ, 2007, 30: 79-84

21 Hashimoto Y, Morimoto T, Fukuyama T. Some speaking plant approach to the synthesis of control system in the greenhouse. Acta Hort, 1985, 174: 219-226

22 Liu X Y, Zhang S M, Wang Y, et al. Characteristics of magnetocardiography and electrocardiography in the time-frequency domain. Chin Sci Bull, 2010, 55: 2091-2098

23 Alan V O, Alan S W. Translated by Liu S T. Signal and System (in Chinese). Xi' an: Xi' an Jiaotong University Press. 2005. 54-256

24 Davies E. New functions for electrical signals in plants. New Phytol, 2004, 161: 607-610

25 Volkov A, Ranatunga D. Plants as environmental biosensors. Plant Signal Behav, 2006, 1: 105-115

26 Gelli A, Blumwald E. Hyperpolarization-activated $\mathrm{Ca}^{2+}$-permeable channels in the plasma membrane of tomato cells. Membr Biol, 1997,
155: $35-45$

27 Volkov A. Green plants: Electrochemical interfaces. Electroanal Chem, 2000, 483: 150-156

28 Volkov A, Brown C. Electrochemistry of plant life. In: Volkov A, ed. Plant Electrophysiology: Theory and Methods. Berlin, Heidelberg: Springer, 2006. 437-459

29 Dziubinska H, Filek M, Koscielniak J, et al. Variation and action potentials evoked by thermal stimuli accompany enhancement of ethylene emission in distant non-stimulated leaves of Vicia faba minor seedlings. Plant Physiol, 2003, 160: 1203-1210

30 Dziubinska H, Trebacz K, Zawadzki T. Transmission route for action potentials and variation potentials in Helianthus аппииs L. Plant Physiol, 2001, 158: 1167-1172

31 Fell J, Elfadil H, Klave R P, et al . Covariation of spectral and nonlinear EEG measures with alpha biofeedback. Int J Neurosci, 2002, 112: $1047-1057$

32 Kang S Z, Cai H J, Feng S Y. Technique innovation and research fields of modern agricultural and ecological water-saving in the future (in Chinese). Trans CSAE, 2004, 20: 1-6

Open Access This article is distributed under the terms of the Creative Commons Attribution License which permits any use, distribution, and reproduction in any medium, provided the original author(s) and source are credited. 